\title{
Post-training unilateral amygdala lesions selectively impair contextual fear memories
}

\author{
Charlotte R. Flavell and Jonathan L.C. Lee ${ }^{1}$ \\ University of Birmingham, Edgbaston, Birmingham B15 2TT, United Kingdom
}

\begin{abstract}
The basolateral amygdala (BLA) and the dorsal hippocampus (dHPC) are both structures with key roles in contextual fear conditioning. During fear conditioning, it is postulated that contextual representations of the environment are formed in the hippocampus, which are then associated with foot shock in the amygdala. However, it is not known to what extent a functional connection between these two structures is required. This study investigated the effect on contextual and cued fear conditioning of disconnecting the BLA and AHPC, using asymmetrically placed, excitotoxic unilateral lesions. Post-training lesions selectively impaired contextual, but not cued, fear, while pretraining lesions resulted in a similar but nonsignificant pattern of results. This effect was unexpectedly observed in both the contralateral disconnection group and the anticipated ipsilateral control, which prompted further examination of individual unilateral lesions of BLA and dHPC. Post-training unilateral dHPC lesions had no effect on contextual fear memories while bilateral dHPC lesions and unilateral BLA lesions resulted in a near total abolition of contextual fear but not cued conditioned fear. Again, pretraining unilateral BLA lesions resulted in a strong but nonsignificant trend to the impairment of contextual fear. Furthermore, an analysis of context test-induced Fos protein expression in the BLA contralateral to the lesion site revealed no differences between post-training SHAM and unilateral BLA lesioned animals. Therefore, post-training unilateral lesions of the BLA are sufficient to severely impair contextual, but not cued, fear memories.
\end{abstract}

In Pavlovian fear conditioning a neutral conditioned stimulus (CS), such as a tone, is paired with an aversive unconditioned stimulus (US), usually a brief foot shock. As a result, a CS-US association is formed, such that subsequent presentations of the CS alone are sufficient to elicit behavioral and physiological (conditioned) responses associated with the US. Conditioned freezing is an example of a robust behavioral measure of fear in rodents and is expressed following both a discrete CS cue and/or exposure to the environmental context in which the CS-US association was formed (Blanchard and Blanchard 1969).

The amygdala has been greatly implicated in the associative component of fear conditioning for both discrete and contextual fear conditioning (for review, see Fendt and Fanselow 1999; LeDoux 2000; Maren 2001). However, the source of the afferent sensory information differs between the two settings. In discrete fear conditioning to auditory cues, the sensory information is relayed to the amygdala largely through the auditory medial geniculate nucleus of the thalamus (Iwata et al. 1986; LeDoux et al. 1986a,b, 1990), while the spatial representations necessary for contextual fear conditioning are formed in the hippocampus (Rudy and O'Reilly 1999; Fanselow 2000). It is, therefore, postulated that information flows in a serial manner, with input about discrete cues from the thalamus and contextual cues from the dorsal hippocampus (dHPC) converging with sensory foot shock information in the basolateral amygdala (BLA). Therefore, the BLA (and in particular, the lateral nucleus) is the primary locus of the fear CS-US association.

Previous studies have shown that bilateral BLA lesions lead to impairment in freezing to both contextual and auditory cues (Phillips and LeDoux 1992; Maren et al. 1996; Cousens and Otto 1998; Goosens and Maren 2001), but see Selden et al. (1991). This effect can be observed when lesions are made both before

\footnotetext{
${ }^{1}$ Corresponding author.

E-mail j.I.c.lee@bham.ac.uk.

Article is online at http://www.learnmem.org/cgi/doi/10.1101//m.025403.111.
}

and after training has occurred (Cousens and Otto 1998), indicating that this structure is essential for both acquisition and recall of the CS-US association. In contrast, post-training bilateral dHPC lesions severely impair freezing to a fear-conditioned context but do not impair freezing to discrete cues (Phillips and LeDoux 1992; Kim et al. 1993; Maren et al. 1997, 1998), supporting a selective role for the dHPC in the spatial representational element of contextual fear conditioning.

Although the interpretation of independent bilateral lesions to the BLA and AHPC indicates that both nuclei are required for contextual fear conditioning, it is not known whether they truly operate in a serial manner as suggested by their hypothesized functional roles. The present study initially employed an anatomical disconnection procedure (e.g., Ferreira et al. 2008; Jimenez and Maren 2009; Bortolanza et al. 2010; Markham et al. 2010) to test directly whether the serial flow of information from the dHPC to the BLA is required selectively for contextual fear conditioning. This involves creating unilateral lesions which target the BLA and the dHPC on opposite hemispheres (CONTRA). Anatomically, the AHPC and the BLA are connected via an almost entirely ipsilateral, bidirectional connection with little or no contralateral connectivity (for review, see McDonald 1998; Pitkanen et al. 2000). Thus, contralateral lesions render the serial flow of information between these two structures impossible. Unilateral lesions in the same hemisphere (IPSI) leave the connection between the BLA and the dHPC intact, thus acting as a control for combined lesion effects. However, we unexpectedly observed an equal deficit in contextual fear conditioning in both the CONTRA and the IPSI groups, which was replicated by unilateral lesions to the BLA alone.

\section{Results}

Histological analysis

Figure 1 illustrates the smallest (black) and most extensive (grey) lesion sites included in the IPSI, CONTRA, unilateral BLA (Uni-BLA), 


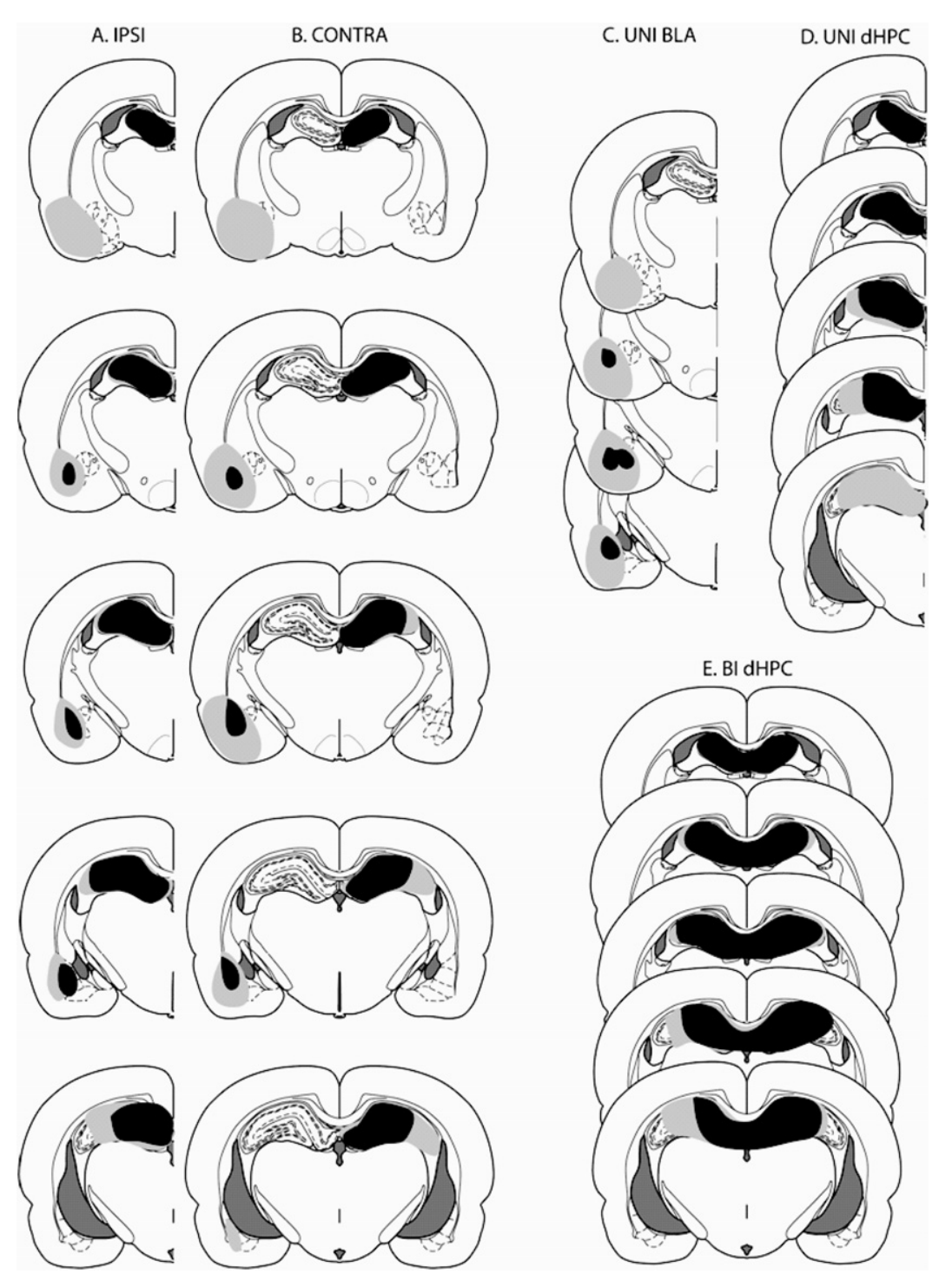

Figure 1. Lesion sites in the amygdala and dorsal hippocampus. Schematic representations of the most (grey shaded) and least (black) extensive included lesions in animals that received IPSI $(A)$, CONTRA $(B)$, UNI BLA $(C)$, UNI dHPC $(D)$, and BI dHPC $(E)$ lesions. (Images adapted from Paxinos and Watson [2007], with permission from Elsevier Ltd. (C) 2007.)

unilateral dHPC (Uni-dHPC), and bilateral dHPC (Bi-dHPC) conditions (Fig. 1A-E, respectively). Figure 2 depicts example photomicrographs of typical lesions in the dHPC and the BLA (Fig. 2B,C, respectively). The contralateral (nonlesioned) side of the same animal are shown as a comparison (Fig. 2A,D, respectively). Animals whose lesion sites were incomplete or absent in one or more of the sites were excluded from further analysis $(n=81)$.

\section{Experiment 1: Both IPSI and CONTRA lesions of the $\mathrm{AHPC}$ and BLA impair freezing to context}

Initial experiments investigated the functional disconnection of BLA and dHPC by using asymmetrically placed unilateral lesions (CONTRA). The effect of these lesions upon freezing was compared to animals with unilateral lesions of the BLA and dHPC on the same side (IPSI), thus leaving one hemisphere intact, and animals that had been infused with PBS (SHAM). Both IPSI and CONTRA SHAM lesions were performed, but, as no statistical dif- ferences were observed between these two groups, the data were combined to form one SHAM group. Similarly, the laterality of the injection sites was counterbalanced across all groups. No statistical differences were observed between injections into the left or right hemispheres so these were also collapsed into one group. Surgery was performed both before (PRE) and after (POST) fear conditioning, to distinguish between impairments in the acquisition or expression of conditioned fear memory. A two-way ANOVA with lesion as the betweensubjects factor and tone as the withinsubjects factor revealed that animals in the PRE and POST groups both demonstrated within-session learning during fear conditioning, as revealed by a main effect of tone (PRE: $F_{(5,125)}=66.47, P<$ 0.001 [Fig. 3A]; POST: $F_{(5,140)}=161.3$, $P<0.001$ [Fig. 3B]). However, given that there was no effect of lesion (PRE: $F_{(2,25)}=0.381, P=0.687$; POST: $F_{(2,28)}=$ $0.468, P=0.631)$ and no interaction (PRE: $F_{(10,125)}=0.946, P=0.494$; POST: $\left.F_{(10,140)}=1.053, P=0.403\right)$, both groups were able to acquire similar levels of freezing across all lesion conditions.

During the context re-exposure test, one-way ANOVA revealed a significant impairment in freezing in the POST $\left(F_{(2,28)}=32.49, P<0.001\right)$ (Fig. 3D), but not PRE $\left(F_{(2,25)}=2.969, P=0.070\right)$ (Fig. $3 \mathrm{C})$ training groups. Post hoc comparisons of the POST groups confirmed that both CONTRA and IPSI lesions reduced expression of the fear memory $(P<0.05)$ compared to SHAM lesions and that there were no significant differences between IPSI and CONTRA lesions $(P>$ 0.05). Although there was no significant effect of PRE IPSI or CONTRA lesions, there was a trend towards decreased freezing levels in both groups compared to sham-lesioned controls.

IPSI and CONTRA lesions conferred no impairment of freezing to the CS in either the PRE $\left(F_{(2,25)}=0.302, P=0.742\right)$ (Fig. 3E) or POST groups $\left(F_{(2,17)}=0.250, P=0.782\right)$ (Fig. $\left.3 \mathrm{~F}\right)$ compared to SHAM.

\section{Experiment 2: Unilateral BLA lesions impair freezing to context}

The common impairment in the IPSI and CONTRA groups may have been due to the unilateral lesion to the BLA or dHPC, or due to a combination of the two, irrespective of their hemispheric localization. In order to disambiguate these possibilities, we studied the effect of unilateral BLA and dHPC lesions. As with the disconnection lesions, unilateral BLA or SHAM lesions were performed either before (PRE) or after (POST) fear conditioning. In contrast, as pretraining bilateral excitotoxic dHPC lesions are generally ineffective against fear conditioning, we only performed post-training dHPC lesions.

For the unilateral BLA lesions, both groups had acquired similar levels of freezing at the end of fear conditioning (Fig. 4A,B), 

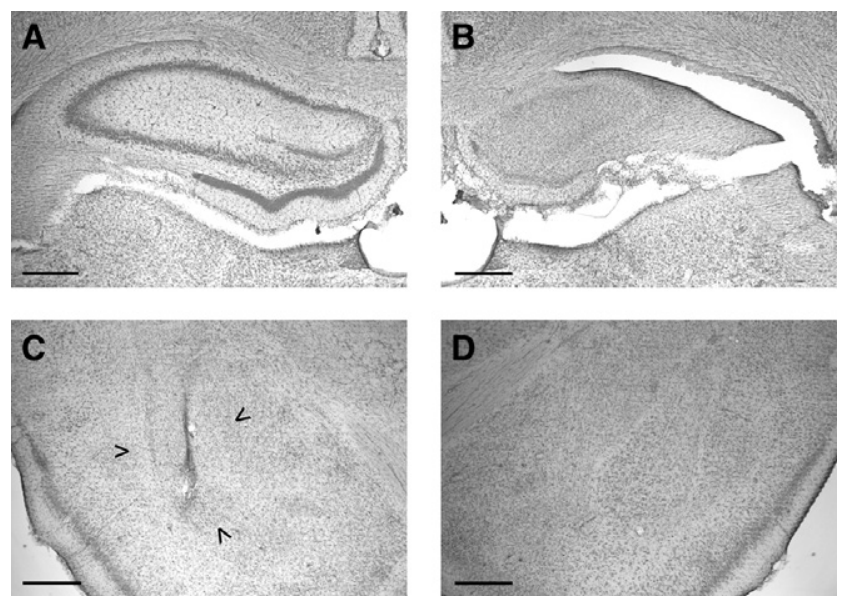

Figure 2. Example photomicrographs of lesions in the BLA and the dHPC. Photomicrographs of representative injection sites (stained with cresyl violet) depicting the nonlesioned $(A)$ and lesioned (B) dHPC, as well as lesioned $(C)$ and nonlesioned (D) BLA. Nonlesioned sections are of the side contralateral to the lesion in the same animal. The arrows in $C$ represent the extent of the lesion site. Scale $=500 \mu \mathrm{m}$.

with a main effect of tone (PRE: $F_{(5,90)}=69.28, P<0.001$; POST: $\left.F_{(5,85)}=73.18, P<0.001\right)$, indicating within-session learning. In the POST groups, there was no effect of lesion $\left(F_{(1,19)}=1.51, P=\right.$ $0.236)$ or tone $\times$ lesion interaction $\left(F_{(5,85)}=2.126, P=0.07\right)$, indicating that the lesion had no effect. However, in the PRE groups there was a significant effect of lesion $\left(F_{(1,18)}=5.14, P=0.036\right)$ and a significant tone $\times$ lesion interaction $\left(F_{(5,90)}=4.11 ; P=\right.$ 0.002). Post-hoc tests $(P<0.05$, Bonferroni corrected) revealed an effect of the BLA lesion at tones 2 and 3 but no effect at tones 1,4 , and 5 . Therefore, while there was evidence that BLA lesions retarded learning, both groups reached equivalent levels of conditioned fear by the end of training.

At the context re-exposure test, a significant impairment in freezing was seen in animals in the POST-Uni BLA group $\left(F_{(1,19)}=11.89, P<0.001\right)$ (Fig. $\left.4 \mathrm{D}\right)$. As with the PRE-IPSI and PRE-CONTRA groups, there was a trend towards lower freezing in the PRE-Uni BLA group compared to the PRE-SHAM group; however, this was not a statistically significant difference $\left(F_{(1,18)}=1.456, P=0.053\right)$ (Fig. $\left.4 \mathrm{C}\right)$. We observed that the failure of the pretraining unilateral BLA lesion to impair contextual freezing significantly was due to a differing variability of effect compared to the POST-Uni BLA group. Comparing the two lesion groups directly, while there was no difference in the levels of contextual freezing $\left(F_{(1,21)}=2.645, P=0.119\right)$, there was a significant difference in variance between the groups $(\mathrm{PRE}=601.2$; POST $=$ 61.5 ; Levene's test: $\left.F_{(1,21)}=11.592, P=0.003\right)$. In contrast, comparison of the PRE and POST unilateral SHAM groups revealed no difference in variance $(\mathrm{PRE}=875.6$; $\mathrm{POST}=669.52$; Levene's test: $\left.F_{(1,16)}=0.118, P=0.735\right)$.

As with the disconnection lesions, no statistically significant differences in auditory fear memory were noted between any of the groups (PRE: $F_{(1,18)}=1.368, P=0.193$; POST: $F_{(1,7)}=1.397$, $P=0.806$ ) (illustrated in Fig. 4E,F).

The effects of bilateral and unilateral dHPC lesions were also investigated. Figure 5A illustrates fear conditioning in the SHAM,
A PRE - Conditioning

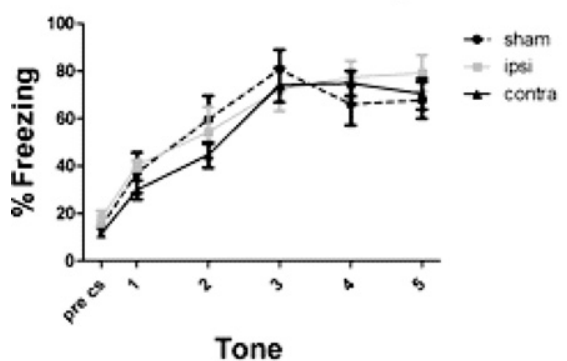

B POST - Conditioning

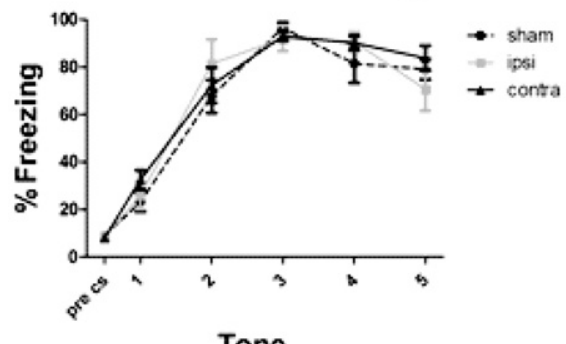

Tone
C PRE - Context

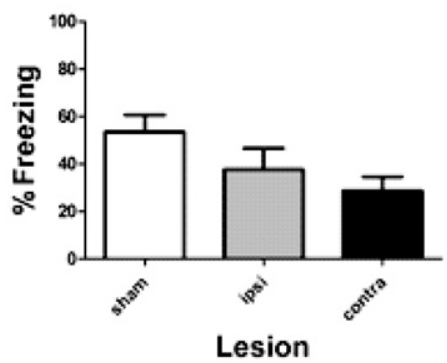

D POST - Context

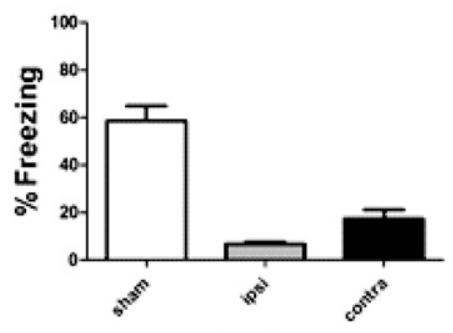

Lesion

\section{E PRE - Freezing to first tone}

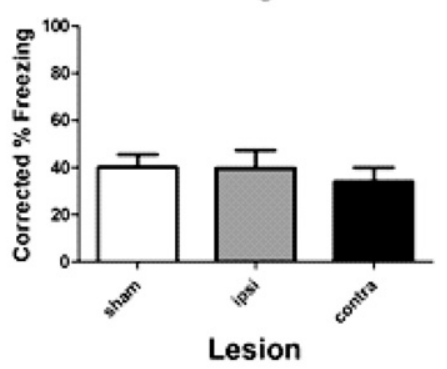

F POST - Freezing to first tone

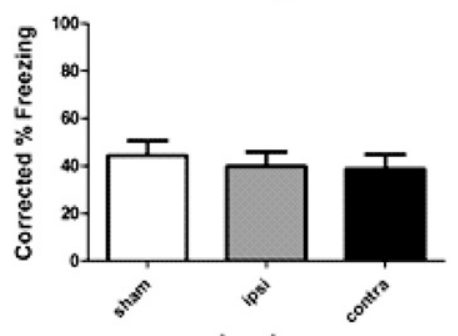

Lesion

Figure 3. Freezing following disconnection lesions to the BLA and the dHPC. Percentage time spent freezing (mean \pm SEM) during fear conditioning, context re-exposure, and CS re-exposure in animals that had received either ipsilateral (IPSI; same hemisphere), contralateral (CONTRA; opposite hemisphere), or sham lesions in the dHPC and BLA before (PRE) or after (POST) fear conditioning. $(A)$ Fear conditioning in rats with pretraining lesions $(n=9$, 10 , and 9$)$. (B) Fear conditioning in rats with post-training lesions $(\mathrm{n}=8,11$, and 12$)$. (C) Context re-exposure in rats with pretraining lesions $(n=9,10$, and 9). (D) Context re-exposure in rats with post-training lesions $(n=8,11$, and 12$)$. ( $E$ ) CS re-exposure in rats with pretraining lesions $(n=9,10$, and 9$)$. $(F) C S$ re-exposure in rats with post-training lesions $(n=6,7$, and 7$)$. To correct for individual differences in pre-CS freezing during the tone test, data are represented as \% time freezing during CS1 minus \% time freezing during the pre-CS period (corrected \% freezing). All animals achieved a similar level of freezing during fear conditioning. Both IPSI and CONTRA lesions impaired freezing to contextual but not auditory cues in POST animals. PRE lesions had no effect. No differences were observed during the CS test. Data presented as mean + SEM. 
A PRE - Conditioning

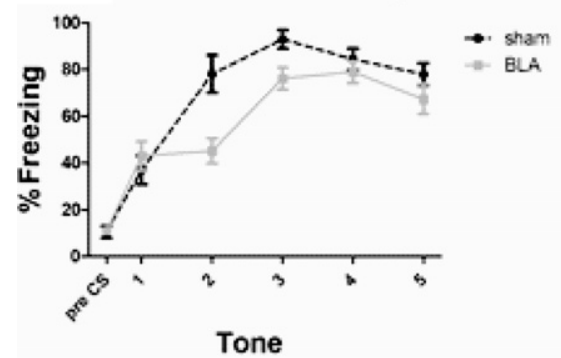

B POST - Conditioning

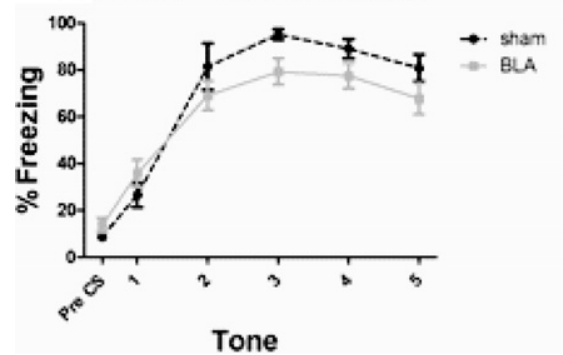

C PRE - Context

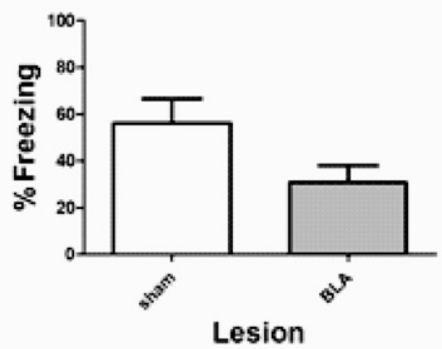

D POST - Context

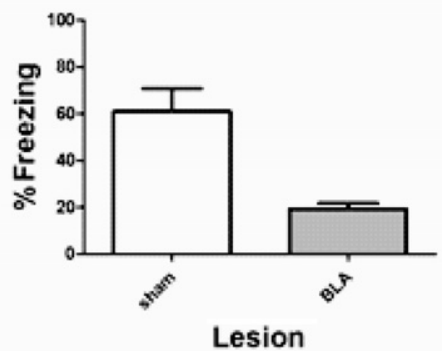

E PRE - Freezing to first tone

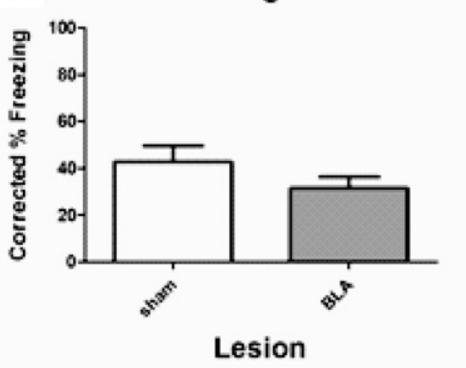

F POST - Freezing to first tone

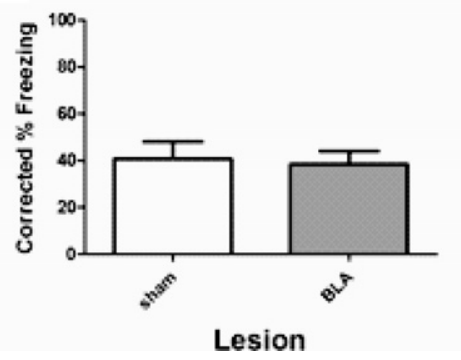

Figure 4. Freezing following unilateral lesions in the BLA. Percentage time spent freezing (mean \pm SEM) during fear conditioning, context re-exposure, and CS re-exposure in animals which had received either unilateral BLA or sham lesions before (PRE) or after (POST) fear conditioning. ( $A$ ) Fear conditioning in rats with pretraining lesions $(n=12$ and 8$)$. (B) Fear conditioning in rats with post-training lesions $(n=11$ and 10$)$. (C) Context re-exposure in rats with pretraining lesions $(n=12$ and 8). (D) Context re-exposure in rats with post-training lesions $(n=11$ and 10$)$. (E) CS re-exposure in rats with pretraining lesions $(n=12$ and 8$)$. ( $F)$ CS re-exposure in rats with post-training lesions ( $n=5$ and 4$)$. To correct for individual differences in pre-CS freezing during the tone test, data are represented as \% time freezing during CS1 minus \% time freezing during the pre-CS period (corrected \% freezing). All animals achieved a similar level of freezing during fear conditioning. Unilateral BLA lesions impaired freezing to contextual but not auditory cues in POST animals. No differences were observed during the CS test. Data presented as mean + SEM.

unilateral dHPC, and bilateral dHPC lesion groups. Within-session learning was indicated by a significant main effect of tone $\left(F_{(5,115)}=129.6 ; P<0.001\right)$, with no effect of lesion $\left(F_{(2,23)}=\right.$ $1.026 ; P=0.318)$ and no tone $\times$ lesion interaction $\left(F_{(10,115)}=\right.$ $0.704 ; P=0.719)$. A one-way ANOVA of the context test data revealed that post-training bilateral dHPC lesions impaired contextual fear memory expression, whereas unilateral dHPC lesions were without effect $\left(F_{(2,23)}=26.61 ; P<0.001\right)$ (Fig. 5B). Post-hoc comparisons $(P<0.05)$ confirmed that freezing levels in the bilateral dHPC lesion group were lower than those in both the sham and unilateral dHPC lesion groups, with the latter groups not differing from each other. This lack of effect following unilateral dHPC lesions indicates that the impairment in freezing seen following IPSI and CONTRA lesions was due solely to the unilateral BLA lesions.

Experiment 3: Fos expression is not affected in contralateral BLA

Experiments 1 and 2 concluded that unilateral BLA lesions led to a deficit in the expression of conditioned contextual fear memories. As this effect was observed only in post-training lesions, it was
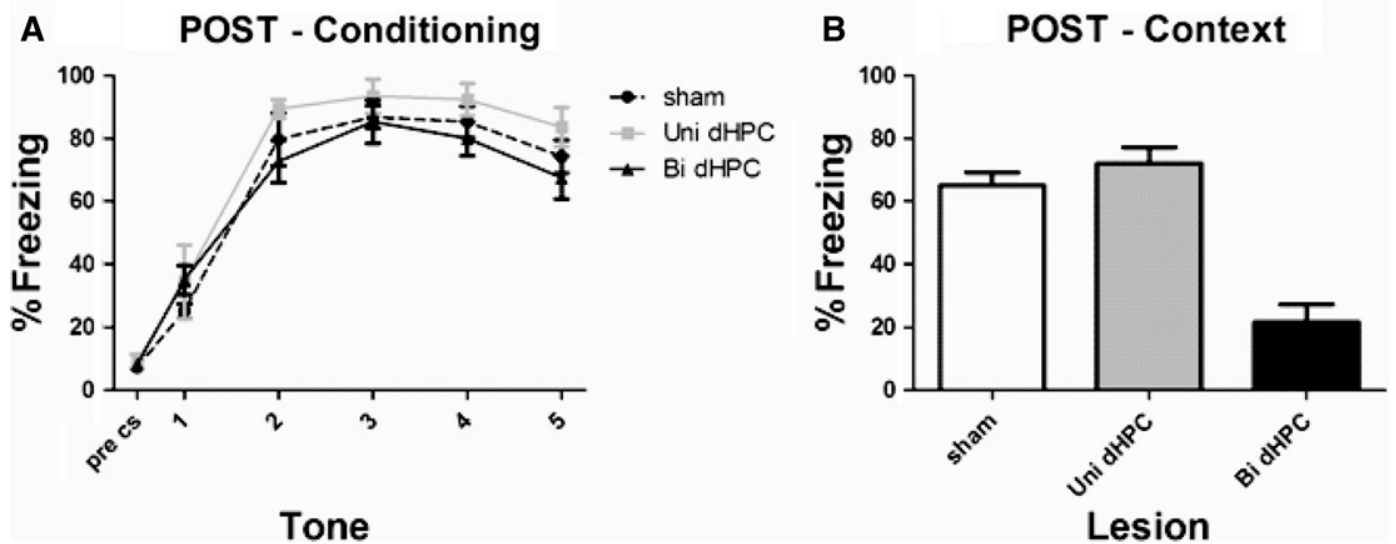

Figure 5. Freezing following unilateral and bilateral lesions in the dHPC. Percentage time spent freezing (mean \pm SEM) during $(A)$ fear conditioning and (B) context re-exposure in animals that had received either unilateral dHPC, bilateral dHPC, or sham lesions after fear conditioning $(n=5,8$, and 13$)$. All animals achieved a similar level of freezing during fear conditioning. Bilateral dHPC lesions conferred a significant impairment in freezing to contextual cues, while unilateral lesions did not. Data presented as mean + SEM. 
unclear as to whether this deficit was due to a genuine unilateral damage or if the contralateral amygdala was also sustaining damage, perhaps due to degeneration of commissural fibers. With this in mind, a subset of rats (SHAM $n=3$, Uni-BLA $n=3$ ) that received fear conditioning and the context test were killed $2 \mathrm{~h}$ following the context test to investigate c-Fos expression in the contralateral amygdala. Fos protein was detected using standard immunohistochemical techniques, and Fos-like immunoreactivity (FLI) in the amygdala contralateral to lesions was counted. Figure 6A depicts FLI in the BLA, and the arrows point to examples of Fos-positive nuclei. There were no differences in FLI between the two groups $\left(F_{(1,4)}=1.439 ; P=0.884\right)$ (Fig. 6B).

\section{Discussion}

The present study has demonstrated that post-training IPSI and CONTRA disconnections of the BLA and the dHPC almost completely eradicated freezing to conditioned contextual stimuli. Furthermore, post-training unilateral lesions of the BLA also resulted in a near complete loss of contextual fear. Post-training lesions to the dHPC only impaired contextual freezing when they were bilateral and not unilateral. In contrast to the profound impact upon contextual freezing, none of the post-training lesions impaired freezing to discrete auditory cues. Moreover, pretraining disconnection and unilateral BLA lesions resulted in a nonsignificant trend to disruption of freezing to contextual but not auditory cues.

An unexpected result was the similar effect of IPSI compared to CONTRA lesions. As one hemisphere is left intact in the IPSI lesion, it was expected to have no effect upon freezing and, therefore, originally intended as a control. The equal impairment of contextual fear observed in the IPSI and CONTRA groups could be due to a mass action effect (Lashley 1931), the magnitude of the behavioral impairment being related simply to the volume of tissue damage, regardless of its location. However, as unilateral BLA lesions were sufficient to cause impairments in contextual freezing, such an explanation appears unlikely. Moreover, the lack of unilateral dHPC lesion effects suggests that the similar deficit in the IPSI and CONTRA groups is due to the common unilateral BLA damage, irrespective of the additional unilateral dHPC lesion.

While much of the literature has focused upon the effect of bilateral amygdala lesions upon conditioned freezing, there is some precedent for the present observation that contextual fear can be disrupted by unilateral lesions conducted after condition-

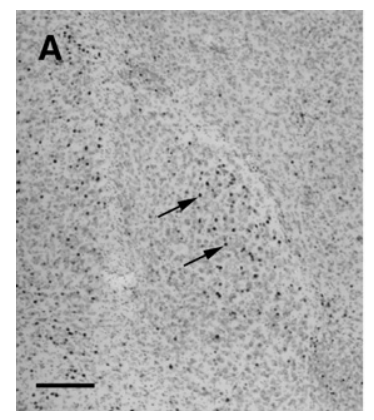

\section{B $\mathrm{FLI}$ in the contralateral BLA}

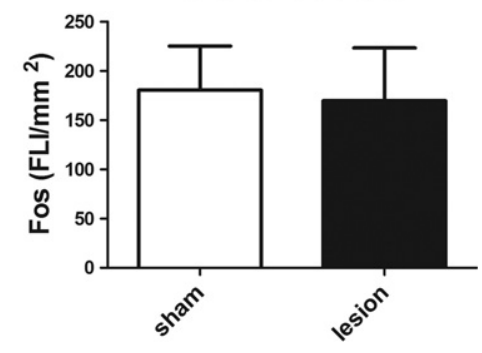

Figure 6. FLI in contralateral BLA. (A) Photomicrograph of Fos-like immunoreactivity (FLI) in the BLA. Arrows indicate examples of what were considered to be Fos-positive nuclei. Scale $=200 \mu \mathrm{m}$. (B) Mean density $( \pm$ SEM) of FLI in the BLA contralateral to lesion sites. No significant differences $(P>0.05)$ were found between SHAM and UNI BLA lesioned animals. Data presented as mean + SEM. ing. Baker and Kim (2004) carried out post-training unilateral electrolytic lesions to the amygdala that were focused upon the BLA and observed impairments in both contextual and discrete cue conditioned freezing. Therefore, by comparison, the present excitotoxic lesions appear to replicate the effect on contextual freezing but not discrete cue freezing. This discrepancy may be related to the differing nature of electrolytic and excitotoxic lesions, especially due to a sparing of axons of passage in the latter (Wallace and Rosen 2001). This has downstream cellular consequences in that there is evidence that immediate-early gene expression varies according to lesion technique (Glenn et al. 2005). However, there is no clear explanation for why this difference in lesion technique should have a particular impact upon discrete cue freezing. Moreover, it was reported that the impairment in contextual freezing was observed only following electrolytic damage to the right and not the left amygdala (Baker and Kim 2004). Here, there was no evidence for laterality in the effects of excitotoxic unilateral lesions to the BLA. Therefore, the relevance of previously observed electrolytic lesion impairments to the present excitotoxic lesion effects remains unclear.

The pretraining unilateral BLA lesion had no statistical impact upon contextual or discrete cue fear conditioning. However, the lesion group displayed a trend towards lower contextual freezing at test than the sham controls $(P=0.053)$. Therefore, there is some evidence that pretraining unilateral BLA lesions impair the acquisition/expression of contextual fear. This is, again, in partial accordance with the previously reported effects of electrolytic lesions. Pretraining electrolytic lesions have resulted in an attenuation, but not complete eradication, of freezing to contextual cues (LaBar and LeDoux 1996; Goosens and Maren 2001; Baker and Kim 2004). However, the effects of electrolytic lesions also extended to discrete cued fear conditioning, whereas there was no sign of a reduction in cued freezing in the present study. The conclusion that pretraining unilateral BLA lesions may impair contextual freezing is further supported by the trends observed in the IPSI and CONTRA lesion groups. It is likely that the similar pattern of results observed in the IPSI, CONTRA, and Uni BLA lesion groups reflects the common unilateral BLA lesion. Pretraining bilateral excitotoxic lesions to the dHPC frequently fail to impair the acquisition and retention of both discrete cued and contextual fear conditioning (Maren et al. 1997; Frankland et al. 1998; Fendt et al. 2005). The former is entirely consistent with the proposed selective role for the hippocampus in contextual processing (Anagnostaras et al. 2001). However, the latter is somewhat paradoxical, and has been explained by the ability of extrahippocampal structures to support contextual processing in the absence of a functional dHPC (Wiltgen et al. 2006). Therefore, it would not be expected that the addition of a unilateral dHPC lesion in either hemisphere would cause an additional impairment to that induced by the unilateral BLA lesion. Indeed, this is exactly what we observed, with the absolute freezing levels in the pretraining unilateral BLA lesion group being very similar to those of the IPSI and CONTRA groups.

There was also evidence that pretraining unilateral BLA lesions retarded the acquisition of conditioned fear. The Uni BLA lesion group was impaired in the acquisition of conditioned freezing to the tone during conditioning. However, this pattern was not replicated in the IPSI and CONTRA groups. Indeed, there was absolutely no evidence for a lesion effect with the addition of a unilateral dorsal hippocampal lesion. There is no reason why the addition of the hippocampal lesion should have reversed the effect of the unilateral BLA lesion. Moreover, none of the pretraining lesion groups showed a deficit in conditioned freezing to the tone at test, which is in accordance with the observation that even the Uni BLA lesion group reached control levels of toneconditioned freezing by the end of the conditioning session. 
Therefore, the reliability and/or interpretative relevance of the apparent deficit in acquisition remain unclear. Moreover, given that the acquisition data are specific for tone fear, it does not impact directly upon the interpretation of the effect of pretraining unilateral lesions upon contextual freezing.

One explanation for why post-training unilateral BLA lesions resulted in a more robust impairment in contextual freezing than did pretraining lesions is the variability of performance at test in the two conditions. In the unilateral BLA lesion groups, there was heterogeneity of variance when the lesion was performed before or after conditioning. In contrast, for the sham controls, there was no heterogeneity of variance with the timing of the lesion. Therefore, it appears that post-training Uni BLA lesions resulted in a decrease of the variability of conditioned freezing relative to SHAMs during the context test. Importantly, the sample sizes in the two conditions were equivalent, indicating that the failure to observe a statistically significant effect in the pretraining condition was not due to a lack of power. Rather, several rats in the pretraining lesion group showed high levels of contextual freezing. In particular, while the maximum level of freezing in the posttraining lesion group was $27.4 \%$, six out of 12 rats exceeded this level in the pretraining condition, with four rats showing freezing levels in excess of $43 \%$. The remaining rats froze at low levels equivalent to the post-training condition. Therefore, it appears that pretraining unilateral BLA lesions impair contextual freezing in some but not all rats. We can only speculate as to the reasons why some rats appear to be resilient to the disruptive effects of the unilateral lesion. However, it may be related to the observation that pretraining bilateral BLA lesions impair contextual fear conditioning in a manner that can be recovered by overtraining (Maren 1999). While the conditioning parameters employed in the current study cannot be described as overtraining, perhaps the mechanisms that allow some rats to compensate for the lack of the BLA unilaterally are similar to those that seemingly allow all rats to recover from the lack of the BLA bilaterally with extended training. Therefore, it might be predicted that the central nucleus of the amygdala, either unilaterally or bilaterally, may support contextual freezing in the unimpaired rats (Zimmerman et al. 2007). Alternatively, it may be that, in the unimpaired rats, the spared unilateral BLA is sufficient to support contextual freezing.

Unilateral BLA interference has been assessed in recent studies that have similarly attempted to define the contribution of serial processing using neuroanatomical disconnection procedures (Jimenez and Maren 2009; Markham et al. 2010). Of particular relevance to the present study is that of Jimenez and Maren (2009), which investigated the functional connection of the BLA and the central nucleus of the amygdala (CeA) using asymmetric lesions. Post-training ipsilateral lesions of BLA and CeA were used for the control group. Although there appeared to be a slight deficit in contextual fear as a result of ipsilateral lesions compared to sham operated controls, it was not statistically significant. Furthermore, they found a much decreased level of freezing in the contra group (Jimenez and Maren 2009). The reason for the discrepancy between these and the current findings are not clear. However, Jimenez and Maren (2009) stressed that the lesions of the BLA and CeA needed to be very small and specific to the target nucleus, so that bilateral lesions were not inadvertently created. Therefore, it is possible that their lesions did not encompass the entire BLA and that the partial rather than complete loss of freezing to contextual cues was an effect of sparing part of the nuclei. Lesions in the current study were all large and usually encompassed most if not all of the lateral, basal, and accessory basal nuclei of the amygdala. While the central nucleus was always spared, other surrounding structures were sometimes, but definitely not consistently, partially lesioned.
A recent study in Syrian hamsters has used post-training, pretest infusions of muscimol reversibly to disconnect the BLA and ventral hippocampus (Markham et al. 2010). In a conditioned defeat setting, unilateral BLA inactivation was found to be sufficient to inhibit memory retrieval. However, it appeared that the unilateral infusion of muscimol had, in fact, caused a bilateral BLA impairment. To explore the effects of the inactivation further, Markham et al. (2010) analyzed the expression of c-Fos. c-Fos is an immediate-early gene expressed following neuronal activation. The protein product (Fos) can be detected using standard immunohistochemical techniques and can be used to reveal multisynaptic pathways of activation (Dragunow and Faull 1989; Kaczmarek 1993). The pattern of Fos-like immunoreactivity revealed a decrease of FLI in the animals that received muscimol, compared to saline. This suggested that unilateral inhibition of the amygdala led to a functional inhibition on the contralateral side, thus mimicking the effects of a bilateral inhibition (Markham et al. 2010). However, in the present study, Fos investigation revealed no differences in FLI in the contralateral BLA of Sham and Uni BLA lesioned animals. This suggests that, in this instance, post-training unilateral lesion of the BLA did not functionally inhibit the contralateral BLA at test. This interpretation is further supported by the finding that the effect of unilateral dHPC lesions on contextual freezing differed completely from that of bilateral dorsal hippocampal lesions. If the unilateral BLA lesion caused behavioral impairments by functionally disrupting the BLA bilaterally, it would be expected that the same pattern would be observed for lesions to the dHPC. Therefore, it is most likely that the effect of unilateral amygdala lesions here is a result of selective disruption of BLA function in one hemisphere alone.

In conclusion, the present results demonstrate that a posttraining unilateral lesion of the BLA is sufficient to impair freezing to conditioned contextual, but not auditory, cues. This effect does not appear to be mediated by a degeneration of the contralateral BLA, as Fos-like immunoreactivity did not differ in the contralateral BLA in either SHAM or Uni BLA animals. This indicates that it is a true unilateral effect and that a bilateral activation of BLA is required for the expression of contextual, but not cued, conditioned fear.

\section{Materials and Methods}

\section{Subjects}

The subjects were 213 experimentally-naive male Lister hooded rats weighing 250-300 g at the beginning of the experiment. Subjects were housed in groups of four in a holding room maintained at $21^{\circ} \mathrm{C}$ on a 12 -h light/dark cycle (lights on at 07:00). Access to food and water was ad libitum throughout, except during behavioral sessions. All procedures complied with the UK 1986 Animals (Scientific Procedures) Act and were performed under project license PPL 40/3205.

\section{Apparatus}

All behavioral training and testing took place in four operant chambers (MedAssociates), each measuring $25 \mathrm{~cm} \times 32 \mathrm{~cm} \times$ $25.5 \mathrm{~cm}$, and housed within a sound-attenuating chamber. Two sides of the operant chambers were constructed of steel, and the ceiling and front and back walls were Perspex, the front also serving as a door. On the side walls were located several modules (retractable levers, LED stimulus lights, food magazine, and auditory stimulus generators). However, the only modules employed during these experiments were the LED house light, located in the top center of the rear wall of each chamber, and the auditory stimulus generator. The grid floors consisted of 19 stainless steel rods (4.8-mm-diameter; $1.6 \mathrm{~mm}$ center-to-center), connected to a shock generator and scrambler (MedAssociates). Below the grid floor was a removable tray. Mounted on the ceiling of the operant 
chamber but within the sound-attenuating chamber was an infrared video camera (Viewpoint Life Sciences).

\section{Surgical procedures}

All animals were anesthetized with a ketamine/medetomidine mixture $\left(60 \mathrm{mg} \mathrm{kg}^{-1} / 0.25 \mathrm{mg} \mathrm{kg}^{-1}\right.$; i.p.) and received perioperative pain relief (buprenorphine, $0.1 \mathrm{mg} \mathrm{kg}^{-1}$; s.c.). Animals were placed into a stereotaxic frame, and a 28-gauge stainless steel cannula was lowered into the basolateral amygdala (BLA) and/ or dorsal hippocampus (dHPC) according to previously published coordinates (Ito et al. 2006). Excitotoxic lesions in the BLA were achieved using 2,3-pyridine dicarbonic acid (quinolinic acid; $0.09 \mathrm{M}$ in phosphate-buffered saline, $\mathrm{pH} 7.4$ [PBS]) and in the dHPC using N-methyl-D-aspartate (NMDA; $0.09 \mathrm{M}$ in PBS). Sham lesions consisted of an infusion of PBS alone. Drugs were drawn up using a Hamilton syringe connected to the cannula via polyelthylene tubing, placed into an infusion pump which delivered drug/saline at a rate of $0.2 \mu \mathrm{L} \mathrm{min}^{-1}$. See Table 1 for stereotaxic coordinates and infusion parameters. Laterality of injection sites was counterbalanced across all groups. Following infusions, the anesthetic was reversed using atipamezole $\left(0.1 \mathrm{mg} \mathrm{kg}^{-1}\right.$; s.c.), and the animals were allowed to recover for $7 \mathrm{~d}$ before beginning/resuming behavioral testing.

\section{Behavioral protocols}

All rats received a fear conditioning session (conditioning) and a context re-exposure session (context). A proportion of rats also received a discrete cue test session (tone). However, due to the investigation of Fos-like immunohistochemistry following the context test, tone test data were not collected in all of the post-training conditions or in the unilateral dHPC and bilateral dHPC lesion groups. Freezing was monitored during all sessions via video cameras, using VideoTrack software (Viewpoint Life Sciences). Fear conditioning was a 15-min session, consisting of a 5-min habituation period, followed by five tone presentations (30 sec, $80 \mathrm{db}$, $5 \mathrm{kHz}$ ), each coterminating with a 1 -sec, $0.5-\mathrm{mA}$ foot shock, with a 90-sec inter-trial interval. Rats underwent surgery either before (PRE) or after (POST) fear conditioning training. Rats in the POST condition that did not exceed $20 \%$ time freezing at the final tone presentation were excluded from the final analysis on the basis that the level of prelesion conditioned fear was insufficient. Context re-exposure occurred either $24 \mathrm{~h}$ after the fear conditioning session (PRE) or $7 \mathrm{~d}$ after surgery (POST) and consisted of a 5 -min re-exposure to the operant box. The tone test occurred 28-30 $\mathrm{h}$ following the context test and was performed inside the same operant box in which conditioning had occurred. To investigate freezing to the tone cue and not contextual cues, the operant boxes were modified by placing a white Perspex insert on the floor of the box, covering the grid floor, a clear Perspex insert with a blue polka dot pattern was placed at the back wall, a few drops of acetic acid were added, and the house light was extinguished for the duration of the session. Animals were placed in the boxes and freezing to the new context was recorded for $5 \mathrm{~min}$. They were then presented with five tones, as described in the conditioning, but no foot shocks were administered.

\section{Immunohistological analysis}

The placement of all lesion sites was verified at the end of the experiment by sectioning the brain into $50-\mu \mathrm{m}$ slices, which were

Table 1. Lesion parameters

\begin{tabular}{|c|c|c|c|c|c|c|}
\hline \multirow[b]{2}{*}{ Site } & \multirow[b]{2}{*}{ Excitotoxin } & \multicolumn{3}{|c|}{$\begin{array}{l}\text { Injection } \\
\text { coordinates }\end{array}$} & \multirow{2}{*}{$\begin{array}{l}\text { Volume } \\
\text { per site } \\
(\mu L)\end{array}$} & \multirow{2}{*}{$\begin{array}{l}\text { Diffusion time } \\
\text { before removal } \\
\text { of injector } \\
(\mathrm{min})\end{array}$} \\
\hline & & AP & ML & DV & & \\
\hline$\overline{\mathrm{dHPC}}$ & NMDA & $\begin{array}{l}-2.8 \\
-4 ?\end{array}$ & $\begin{array}{l} \pm 1.6 \\
+26\end{array}$ & $\begin{array}{l}-3.2 \\
-29\end{array}$ & $\begin{array}{l}0.4 \\
0.4\end{array}$ & 1 \\
\hline BLA & $\begin{array}{l}\text { Quinolinic } \\
\text { acid }\end{array}$ & $\begin{array}{l}-2.8 \\
-3.0\end{array}$ & $\begin{array}{l} \pm 4.5 \\
\pm 4.5\end{array}$ & $\begin{array}{l}-7.5 \\
-7.5\end{array}$ & $\begin{array}{l}0.3 \\
0.2\end{array}$ & $\begin{array}{l}2 \\
2\end{array}$ \\
\hline
\end{tabular}

then stained using cresyl violet. Animals deemed not to have had a successful lesion were excluded from further analysis. This was usually due to either an absent or partial lesion rather than an off-target injection site.

Rats that were included in the c-Fos study (POST Sham $n=3$; POST Unilateral BLA $n=3$ ) were transcardially perfused $2 \mathrm{~h}$ after the context test and stored in 4\% paraformaldehyde in PBS. The brains were subsequently cut into sections $80-\mu \mathrm{m}$ thick, and every fourth section was removed and immunohistochemically processed for Fos-like immunoreactivity using a rabbit anti-Fos primary antibody (Santa Cruz Biotechnology Inc.) and a "Vectastain ABC kit" (Vector Laboratories LTD) containing a biotinylated goat anti-rabbit IgG secondary antibody. In brief, following a 30-min incubation in $0.3 \%$ hydrogen peroxide to remove endogenous peroxidases, the sections were left overnight at $4^{\circ} \mathrm{C}$ in a solution containing 1:5000 primary antibody and 3\% normal goat serum, diluted in $0.1 \%$ Triton X-100 in PBS. The following day, the sections were washed three times in PBS, then incubated for $2 \mathrm{~h}$ at room temperature with the secondary antibody. The $A B C$ reagent supplied in the kit was then applied as per instructions. FLI was visualized, using a DAB (3-3, diaminobenzidine) Peroxidase substrate kit (Vector Laboratories LTD). All sections were then counterstained using cresyl violet.

FLI in the BLA was examined in all sections immunohistochemically processed. Overlapping images of the BLA were obtained using an Olympus BX50 light microscope attached to a Leica DFC425 camera, using Leica Application Suite v3.7 (LAS). Composite high-power images of the entire BLA were created using Adobe Photoshop, which were then transferred back into LAS. The Interactive Measurements module was used to mark the position of each Fos-positive nucleus within the BLA and to gain a measurement of the area of the BLA in that section. The density of FLI was calculated by dividing the total number of Fos-positive neurones in each section by the area of BLA.

\section{Statistical analysis}

Data are presented as mean \pm SEM percentage time freezing. Oneor two-way repeated-measures ANOVAs and unpaired $t$-tests were performed on the data with factors lesion, time of lesion, and test as appropriate. Tukey's multiple comparison test was selected for post-hoc comparisons and Levene's homogeneity of variance test was used to compare variability of freezing across groups. To correct for individual differences in pre-CS freezing, the tone test data are presented as percentage freezing to the first tone presentation minus \% time freezing during the 5 min before tone presentation in the altered context (corrected \% freezing). A significance level of $P<0.05$ was selected for all analyses. Animals in the POST groups which had not demonstrated $\geq 20 \%$ freezing during the last tone of the conditioning session were deemed not to have learned and were excluded from further analysis.

\section{Acknowledgments}

We thank Dave Barber for his expert technical assistance.

\section{References}

Anagnostaras SG, Gale GD, Fanselow MS. 2001. Hippocampus and contextual fear conditioning: Recent controversies and advances. Hippocampus 11: 8-17.

Baker KB, Kim JJ. 2004. Amygdalar lateralization in fear conditioning: Evidence for greater involvement of the right amygdala. Behav Neurosci 118: $15-23$.

Blanchard RJ, Blanchard DC. 1969. Crouching as an index of fear. J Comp Physiol Psychol 67: 370-375.

Bortolanza M, Wietzikoski EC, Boschen SL, Dombrowski PA, Latimer M, Maclaren DA, Winn P, Da Cunha C. 2010. Functional disconnection of the substantia nigra pars compacta from the pedunculopontine nucleus impairs learning of a conditioned avoidance task. Neurobiol Learn Mem 94: 229-239.

Cousens G, Otto T. 1998. Both pre- and post-training excitotoxic lesions of the basolateral amygdala abolish the expression of olfactory and contextual fear conditioning. Behav Neurosci 112: 1092-1103. 
Dragunow M, Faull R. 1989. The use of c-fos as a metabolic marker in neuronal pathway tracing. J Neurosci Methods 29: 261-265.

Fanselow MS. 2000. Contextual fear, gestalt memories, and the hippocampus. Behav Brain Res 110: 73-81.

Fendt M, Fanselow MS. 1999. The neuroanatomical and neurochemical basis of conditioned fear. Neurosci Biobehav Rev 23: 743-760.

Fendt M, Fanselow MS, Koch M. 2005. Lesions of the dorsal hippocampus block trace fear conditioned potentiation of startle. Behav Neurosci 119: $834-838$.

Ferreira TL, Shammah-Lagnado SJ, Bueno OF, Moreira KM, Fornari RV, Oliveira MG. 2008. The indirect amygdala-dorsal striatum pathway mediates conditioned freezing: Insights on emotional memory networks. Neuroscience 153: 84-94.

Frankland PW, Cestari V, Filipkowski RK, McDonald RJ, Silva AJ. 1998. The dorsal hippocampus is essential for context discrimination but not for contextual conditioning. Behav Neurosci 112: 863-874.

Glenn MJ, Lehmann H, Mumby DG, Woodside B. 2005. Differential fos expression following aspiration, electrolytic, or excitotoxic lesions of the perirhinal cortex in rats. Behav Neurosci 119: 806-813.

Goosens KA, Maren S. 2001. Contextual and auditory fear conditioning are mediated by the lateral, basal, and central amygdaloid nuclei in rats. Learn Mem 8: 148-155.

Ito R, Robbins TW, McNaughton BL, Everitt BJ. 2006. Selective excitotoxic lesions of the hippocampus and basolateral amygdala have dissociable effects on appetitive cue and place conditioning based on path integration in a novel Y-maze procedure. Eur J Neurosci 23: $3071-3080$

Iwata J, LeDoux JE, Meeley MP, Arneric S, Reis DJ. 1986. Intrinsic neurons in the amygdaloid field projected to by the medial geniculate body mediate emotional responses conditioned to acoustic stimuli. Brain Res 383: $195-214$.

Jimenez SA, Maren S. 2009. Nuclear disconnection within the amygdala reveals a direct pathway to fear. Learn Mem 16: 766-768.

Kaczmarek L. 1993. Molecular biology of vertebrate learning: Is c-fos a new beginning? J Neurosci Res 34: 377-381.

Kim JJ, Rison RA, Fanselow MS. 1993. Effects of amygdala, hippocampus, and periaqueductal gray lesions on short- and long-term contextual fear. Behav Neurosci 107: 1093-1098.

LaBar KS, LeDoux JE. 1996. Partial disruption of fear conditioning in rats with unilateral amygdala damage: Correspondence with unilateral temporal lobectomy in humans. Behav Neurosci 110: 991-997.

Lashley KS. 1931. Mass action in cerebral function. Science 73: 245-254.

LeDoux JE. 2000. Emotion circuits in the brain. Annu Rev Neurosci 23: $155-184$.

LeDoux JE, Iwata J, Pearl D, Reis DJ. 1986a. Disruption of auditory but not visual learning by destruction of intrinsic neurons in the rat medial geniculate body. Brain Res 371: 395-399.

LeDoux JE, Sakaguchi A, Iwata J, Reis DJ. 1986b. Interruption of projections from the medial geniculate body to an archi-neostriatal field disrupts the classical conditioning of emotional responses to acoustic stimuli. Neuroscience 17: 615-627.
LeDoux JE, Farb C, Ruggiero DA. 1990. Topographic organization of neurons in the acoustic thalamus that project to the amygdala. J Neurosci 10: 1043-1054.

Maren S. 1999. Neurotoxic basolateral amygdala lesions impair learning and memory but not the performance of conditional fear in rats. J Neurosci 19: 8696-8703.

Maren S. 2001. Neurobiology of Pavlovian fear conditioning. Annu Rev Neurosci 24: 897-931.

Maren S, Aharonov G, Fanselow MS. 1996. Retrograde abolition of conditional fear after excitotoxic lesions in the basolateral amygdala of rats: Absence of a temporal gradient. Behav Neurosci 110: $718-726$.

Maren S, Aharonov G, Fanselow MS. 1997. Neurotoxic lesions of the dorsal hippocampus and Pavlovian fear conditioning in rats. Behav Brain Res 88: $261-274$

Maren S, Anagnostaras SG, Fanselow MS. 1998. The startled seahorse: Is the hippocampus necessary for contextual fear conditioning? Trends Cogn Sci 2: 39-42.

Markham CM, Taylor SL, Huhman KL. 2010. Role of amygdala and hippocampus in the neural circuit subserving conditioned defeat in Syrian hamsters. Learn Mem 17: 109-116.

McDonald AJ. 1998. Cortical pathways to the mammalian amygdala. Prog Neurobiol 55: 257-332.

Paxinos G, Watson C. 2007. The rat brain in stereotaxic coordinates. Academic Press, London.

Phillips RG, LeDoux JE. 1992. Differential contribution of amygdala and hippocampus to cued and contextual fear conditioning. Behav Neurosci 106: $274-285$.

Pitkanen A, Pikkarainen M, Nurminen N, Ylinen A. 2000. Reciprocal connections between the amygdala and the hippocampal formation, perirhinal cortex, and postrhinal cortex in rat. A review. Ann N Y Acad Sci 911: 369-391.

Rudy JW, O'Reilly RC. 1999. Contextual fear conditioning, conjunctive representations, pattern completion, and the hippocampus. Behav Neurosci 113: 867-880.

Selden NR, Everitt BJ, Jarrard LE, Robbins TW. 1991. Complementary roles for the amygdala and hippocampus in aversive conditioning to explicit and contextual cues. Neuroscience 42: 335-350.

Wallace KJ, Rosen JB. 2001. Neurotoxic lesions of the lateral nucleus of the amygdala decrease conditioned fear but not unconditioned fear of a predator odor: Comparison with electrolytic lesions. J Neurosci 21: 3619-3627.

Wiltgen BJ, Sanders MJ, Anagnostaras SG, Sage JR, Fanselow MS. 2006. Context fear learning in the absence of the hippocampus. J Neurosci 26: 5484-5491.

Zimmerman JM, Rabinak CA, McLachlan IG, Maren S. 2007. The central nucleus of the amygdala is essential for acquiring and expressing conditional fear after overtraining. Learn Mem 14: 634-644.

Received December 22, 2011; accepted in revised form February 22, 2012. 


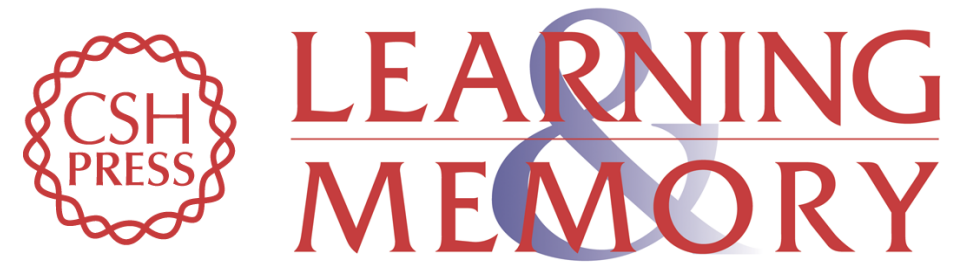

\section{Post-training unilateral amygdala lesions selectively impair contextual fear memories}

Charlotte R. Flavell and Jonathan L.C. Lee

Learn. Mem. 2012, 19:

Access the most recent version at doi:10.1101/Im.025403.111

References This article cites 38 articles, 9 of which can be accessed free at:

http://learnmem.cshlp.org/content/19/6/256.full.html\#ref-list-1

License

Email Alerting Receive free email alerts when new articles cite this article - sign up in the box at the Service top right corner of the article or click here. 